\title{
Clinical applications of the Scheimpflug principle in Ophthalmology
}

\author{
Aplicações clínicas do princípio de \\ Scheimpflug na Oftalmologia
}

Fernando Faria-Correia', Renato Ambrósio Jr. ${ }^{2}$

\begin{abstract}
This article presents a review of the principles and clinical applications of the Scheimpflug principle in the anterior segment imaging. By providing a three-dimensional image of the anterior segment, this technology provides elevation and curvature data of the anterior and posterior surfaces of the cornea, pachymetric mapping, the total refractive power of the cornea and the anterior segment biometry. For the refractive surgery sub-specialty, this approach improves the ability to identify cases at risk of ectasia, as well as the planning and evaluation of the results of surgical procedures. Recently, this technology was introduced in corneal biomechanical in vivo evaluations and in femtosecond laser-assisted cataract surgery.
\end{abstract}

Keywords: Scheimpflug; Tomography; Biomechanics; Cornea; Cataract; Refractive surgery

\section{RESUMO}

Este artigo apresenta uma revisão dos princípios e das aplicações clínicas do princípio de Scheimpflug na área da imagiologia do segmento anterior. Ao disponibilizar uma imagem tridimensional do segmento anterior, esta tecnologia permite a caraterização da elevação e curvatura das superfícies anterior e posterior da córnea, o mapeamento paquimétrico, o cálculo do poder refrativo total da córnea e a biometria do segmento anterior. Na subespecialidade de cirurgia refrativa, esta abordagem melhora a capacidade de identificação de casos com risco de desenvolver ectasia, bem como de planeamento e de avaliação dos resultados dos procedimentos cirúrgicos. Recentemente, esta tecnologia foi introduzida na avaliação biomecânica in vivo da córnea e na cirurgia de catarata assistida por laser de femtossegundo.

Descritores: Scheimpflug; Tomografia; Biomecânica; Córnea; Catarata; Cirurgia refrativa

\footnotetext{
1 Escola das Ciências da Saúde, Universidade do Minho, Braga, Portugal; Study Group on Corneal Tomography and Biomechanics of Rio de Janeiro, Rio de Janeiro, RJ, Brazil.

${ }^{2}$ Universidade Federal de São Paulo, São Paulo, SP, Brazil; Study Group on Corneal Tomography and Biomechanics of Rio de Janeiro, Rio de Janeiro, RJ, Brazil
}

The authors declare no conflicts of interests.

Received for publication 10/07/2015 - Accepted for publication 07/10/2015 


\section{INTRODUCTION}

A dvances in diagnostic capabilities have been critical to the evolution of refractive surgery, which emerged as a new subspecialty in the early 1980 s. ${ }^{1}$ Improving imaging methods of the cornea and the anterior segment is related to the continuous need to increase the safety and effectiveness of surgical procedures. ${ }^{2}$ Linked to a better selection of candidates for refractive surgery, the development of diagnostic technologies dramatically favored surgical planning capabilities, including personalization of laser ablation treatment and the evaluation of results and complications of these procedures. ${ }^{3-8}$ This knowledge also had an impact on the selection of the type and power of the intraocular lens to be implanted in the cataract surgery. ${ }^{9,10}$ In addition, the treatment of complex cases such as keratoconus, corneal dystrophies and other causes of irregular astigmatism also has developed due to advances in the imaging of the cornea and anterior segment. ${ }^{11-15}$

Initially, the main obstacle was the limitations inherent in the computer technology. The technological development has allowed the acquisition and analysis of images, having been key to the development of corneal topography. Stephen Klyce, PhD, is known for having developed derivative color maps of quantitative analysis of various points of the corneal curvature maps. ${ }^{16}$ The analysis of the reflected images of the Placido's disk has been the dominant technique for the analysis of the anterior corneal surface. ${ }^{2}$ Alternatively, the scan photogrammetry uses a stereo triangulation technique wherein a regular pattern consisting of horizontal and vertical lines is projected onto the eye surface to reconstruct the elevation of the anterior corneal surface. ${ }^{17}$ Michael Belin, MD, developed the basis for calculating the elevation maps in relation to a reference surface being defined by a geometric shape (spheric, aspheric or toric ellipsoid) which best fits the actual corneal surface. ${ }^{17,18}$ ENREF_17 Both the Placido's disk and the scan photogrammetry are able to calculate the maps of axial (sagittal) and tangential (instantaneous) curvature of elevation and refraction of the anterior corneal surface. Other systems, such as the Hartmann-Shack sensor for analysis of front-of-wave, also incorporate a device for corneal topography analysis. ${ }^{2}$ However, these devices are limited to the analysis of the anterior corneal surface. ${ }^{19}$

Regarding the measurement of the thickness of the cornea, the optical pachymeter was presented by David Maurice, PhD in 1955. ${ }^{20}$ The ultrasonic technique has demonstrated greater repeatability than the optical pachymeter, but only provided data of a single point. ${ }^{19-21}$ Mandell e Polse proposed a study of the horizontal profile of the corneal thickness using a modified optical pachymeter. In this context, the variation of thickness in the horizontal meridian proved to be a feature for the diagnosis of keratoconus. $^{22}$

The development of the anterior segment tomography allowed the assessment of the profile of the corneal thickness from the pachymetry mapping. ${ }^{23,24}$ Tomography (from the Greek: "tomos" means slice and "grafia" means describe) is a concept that represents the three-dimensional reconstruction of the cornea, providing detailed information on the thickness and the anterior and posterior surfaces of the cornea. ${ }^{19}$ Different technologies such as the horizontal slit scanning, the rotating Scheimpflug camera, high frequency ultrasounds, and optical coherence tomography are commercially available from various instruments. ${ }^{19,25}$

Advances in corneal imaging were beyond the tomographic characterization. The concept of customized biomechanics in refractive surgery was introduced by Cynthia Roberts, PhD, in 2005. ${ }^{26}$ The Ocular Response Analyzer (ORA - Reichert Inc., Depew, USA) was introduced as the first device to assess in vivo the biomechanics of the cornea. ${ }^{27,28}$ ORA is a non-contact tonometer (NCT) which uses an electroquantitative system for controlling the deformation of the cornea by the corneal reflection of infrared light. Other technologies, such as the Scheimpflug image and optical

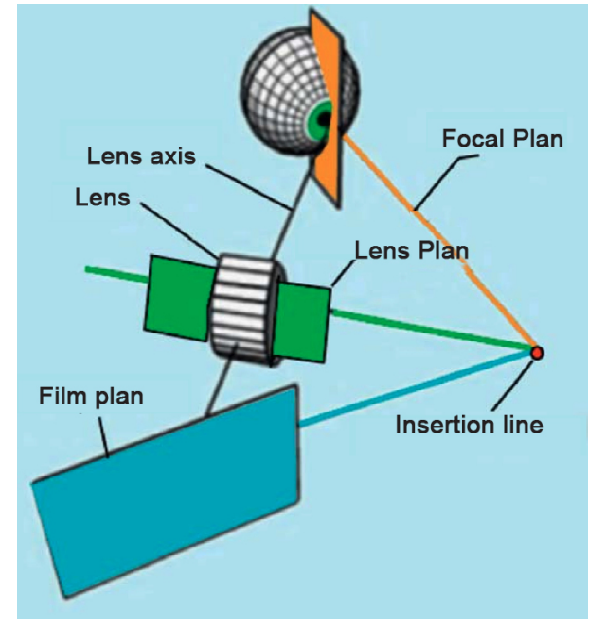

Figure 1: Scheme of the Scheimpflug principle.

coherence tomography, have also been recently introduced to provide dynamic measurements of the corneal deformation. ${ }^{29,30}$ Finally, imaging of the cornea and the anterior segment was also used in cataract surgery assisted by femtosecond laser. ${ }^{31-33}$

This review focuses on the application of the Scheimpflug principle for laser refractive surgery, including its diagnostic capability and biomechanical assessment of the cornea, as well as its recent use in the planning of assisted cataract surgery by femtosecond laser.

\section{Scheimpflug Principle}

The Scheimpflug principle is a geometric rule commonly used in photography. This concept was first described by Jules Carpentier in 1901, having been cited and credited in the original patent by Theodor Scheimpflug in $1904 .{ }^{34}$ In this technique, three imaginary planes - the film plane, the lens plane and the focal plane - are arranged in a non-parallel way (Figure 1). The lens is tilted so that the resulting plane of the lens intercept the planes of the film and focus in a line of intersection known as Scheimpflug line. In a typical photographic camera, the film plane and the lens plane are parallel to each other, and also relative to the focal plane. This principle allows increasing the depth of focus and sharpness of image points located on different planes. $^{34}$

Scheimpflug images of the corneal and anterior segment

The Scheimpflug photography was used in the imaging of the anterior segment by devices EAS 1000 by Nidek (Gamagori, Japan) and SL-45 by Topcon (Tokyo, Japan). ${ }^{35,36}$ These systems have the ability to measure the dispersion of light along the optical axis, allowing the detection of changes in the transparency of the lens over time..$^{35}$ Associated to the optical densitometry, recording these images also offer biometric measurements of the anterior segment such as the anterior chamber depth and peripheral angle measurements. ${ }^{37}$ However, these systems did not hold the threedimensional reconstruction of the anterior segment.

In 1995, the optical cross-sectioning for examination of the cornea was first introduced commercially with the Orbscan [(originally Orbtek, Inc.) Bausch \& Lomb Surgical, Salt Lake City, USA]. ${ }^{38-42}$ ENREF_39_ENREF_39 This instrument is designed to provide tomographic data (three-dimensional reconstruction), but the nomenclature regarding the CT concept was not set yet, so that it was still referred as topography. ${ }^{19,38,42}$ This system introduced the slit-scanning imaging technique or the cobblestone methodology, involving the projection of 40 slits (12.50 $\mathrm{mm}$ height and $0.30 \mathrm{~mm}$ wide) with a Scheimpflug angle of 45 degrees. However, Orbscan slit images do not exhibit the same depth of field as compared to those obtained by the Scheimpflug systems (Figure 2). In its first version, the Orbscan 


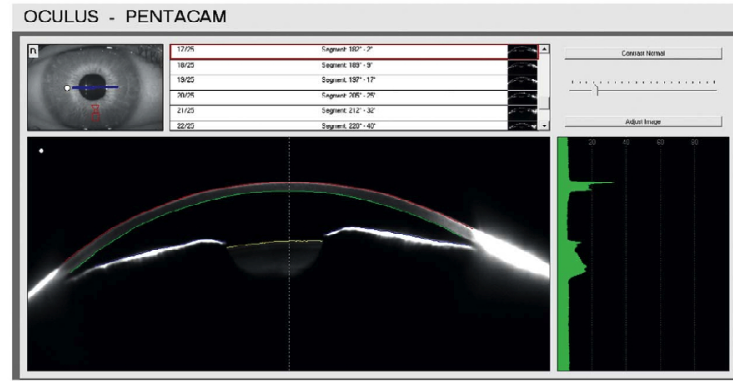

Figure 2: Scheimpflug image of the cornea and anterior segment

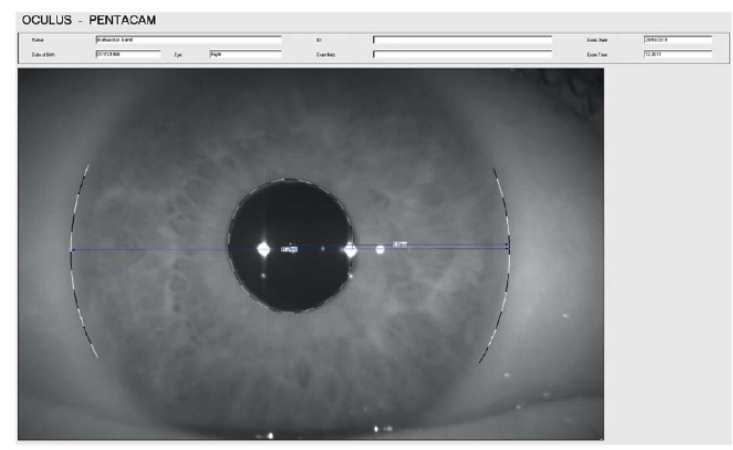

Figure 3: High-resolution front iris camera.

provided information from the anterior curvature extrapolated from elevation data. In 1999, a Placido disk was integrated into the Orbscan II to obtain data directly from the anterior curvature.

The digital tomography with rotating Scheimpflug camera has been recognized as an evolution of the horizontal cross section (cobblestone methodology) in the tomographic assessment of the cornea and anterior segment. Although the capture of horizontal images does not have points in common, the system has a rotating center common to all the images, which makes the registration more precise. ${ }^{43,44}$ The Pentacam (Oculus, Wetzlar, Germany) was the first system available to perform digital tomography of the cornea and anterior segment using rotational
Scheimpflug photography. This device was introduced in 1999, and introduced commercially in 2002. Pentacam, along with the rotating Scheimpflug camera, is part of a second front chamber to control the attachment and compensate the ocular alignment.

In the acquisition mode, optimal alignment is obtained with the first Purkinje reflection of the cornea using both the front chamber and the Scheimpflug one before automatically initiating the exam. A second high-resolution front camera records the size and orientation of the pupillary opening, serving as a guide for three-dimensional reconstruction. This camera also provides black-and-white measurements of the pupil size (Figure 3). The analysis of the three-dimensional Scheimpflug image provides data from the anterior and posterior surface of the cornea, anterior surface of the iris and the crystalline. As the system employs blue visible light (wavelength of $475 \mathrm{~nm}$, free from ultraviolet radiation in Pentacam), that is sensitive to corneal opacities, resulting in hyperreflective images of inaccurate contour. Due to total internal reflection in the peripheral cornea, direct visualization of the anterior chamber angle is not possible. However, the extrapolation software is able to provide an estimate of the iris-corneal angle with relatively high accuracy. ${ }^{45}$ Currently, there are other business units that incorporate rotational Scheimpflug imaging technology, and in particular Galilei (Ziemer, Switzerland), TMS-5 (Tomey, Nagoya, Japan); Sirius (CSO; Florence, Italy) and Preciso (Ivis Technologies, Taranto, Italy). Table 1 presents the diagnostic capabilities of all devices with Scheimpflug imaging technology.

\section{Corneal tomography using the Scheimpflug principle for screening ectasia}

One of the most important applications of corneal computed tomography relates to the diagnosis of keratoconus and other ectasia diseases of the cornea. ${ }^{3,46-49}$ Pachymetry and elevation indices proved to be effective to detect keratoconus. ${ }^{50-}$ ${ }_{52}$ The graphics of the spatial profile and the percentage increase in corneal thickness describe the ring pachymetry increase since the thinnest point. ${ }^{23,24,51}$ These charts are available on Pentacam and have been used successfully in the diagnosis of keratoconus. ${ }^{24,50,53}$ Pachymetry progression indices (PPI) are calculated for all semi-meridians of the cornea, such that the average of all meridians (PPI Ave) and the meridian with maximum pachymetry progression (PPI max) are reported. The "Ambrósio Relational Thickness" (ART) parameter is the ratio

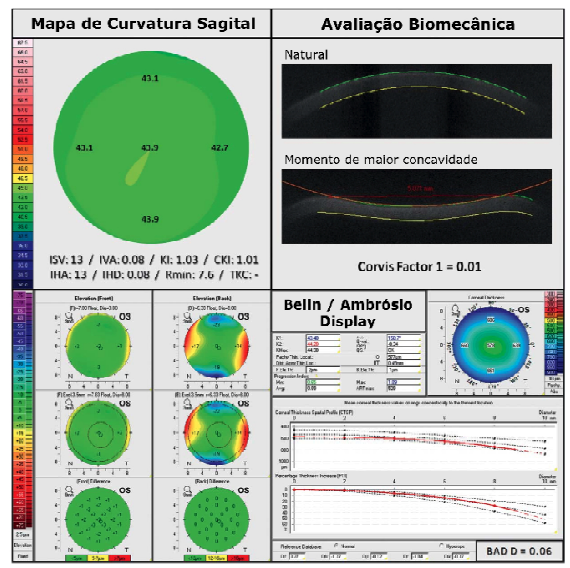

Figure 4: Detailed analysis of a normal cornea, including the map of front curve (sagittal) using the absolute scale of SmolekKlyce, BAD and biomechanical evaluation with CorVIS ST. BAD-D $<1.45$, ART-Max $>412$ e "Corvis Factor $1 "<0.2$ are the most relevant findings. ART, Ambrosio Relational Thickness; BAD, Belin-Ambrosio Enhanced Ectasia Display
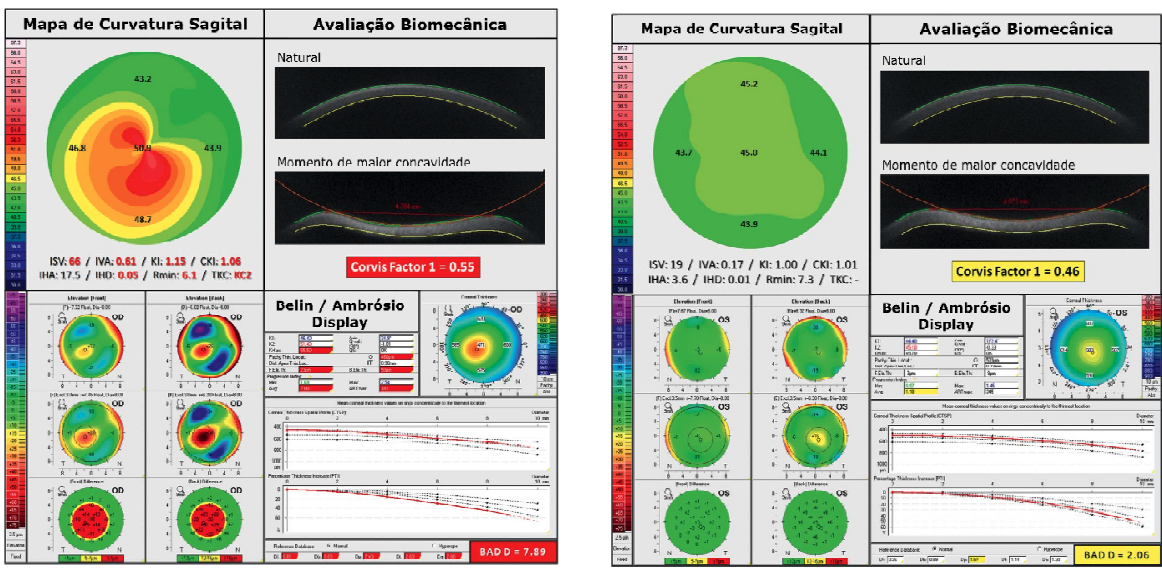

Figure 5: Detailed analysis of both eyes in the same patient with very asymmetrical keratoconus A. Keratoconus in the anterior curvature map, BAD-D $>2.5$, ART-Max $<360$ and "Corvis Factor 1" $>0.35$; B. Forme fruste keratoconus with a relatively normal anterior curvature map, but with BAD-D $>1.45$, ART-Max $<412$ and "Corvis Factor 1" $>0.25$. ART, Ambrosio Relational Thickness; BAD, Belin-Ambrosio Enhanced Ectasia Display 
Table 1

Comparative table of instruments with Scheimpflug image

\begin{tabular}{|c|c|c|c|c|c|c|}
\hline $\begin{array}{l}\text { Company } \\
\text { Photography }\end{array}$ & $\begin{array}{c}\text { Pentacam } \\
\text { Oculus, Germany } \\
\text { Rotational }\end{array}$ & $\begin{array}{c}\text { Galilei } \\
\text { Ziemer, Switzerland } \\
\text { Rotational }\end{array}$ & $\begin{array}{c}\text { TMS-5 } \\
\text { Tomey, Japan } \\
\text { Rotational }\end{array}$ & $\begin{array}{c}\text { Precisio } \\
\text { Ivis, Italy } \\
\text { Rotational }\end{array}$ & $\begin{array}{c}\text { Sirius } \\
\text { CSO, Italy } \\
\text { Rotational }\end{array}$ & $\begin{array}{c}\text { Orbscan } \\
\text { Bausch\&Lomb,USA } \\
\text { Horizontal } \\
\text { cross section }\end{array}$ \\
\hline Placido's disk & No & Yes & Yes & Yes & Yes & Yes \\
\hline Elevation maps & Yes & Yes & Yes & Yes & Yes & Yes \\
\hline $\begin{array}{l}\text { Refractive power } \\
\text { map of the cornea }\end{array}$ & Yes & Yes & Yes & Yes & Yes & Yes \\
\hline $\begin{array}{l}\text { Pachymetric map } \\
\text { Graphic of the space }\end{array}$ & Yes & Yes & Yes & Yes & Yes & Yes \\
\hline profile of the thickness & No & No & No & No & No & No \\
\hline Cataract analysis & Yes & Yes & Yes & No & Yes & Yes \\
\hline $\begin{array}{l}\text { Analysis of the } \\
\text { anterior chamber }\end{array}$ & Sim & Sim & Sim & Sim & Sim & Sim \\
\hline
\end{tabular}

Table 2

Results of the curves "receiver operating characteristic" (ROC) of the parameters of Pentacam (331 normal patients vs. 242 patients with bilateral clinical keratoconus)

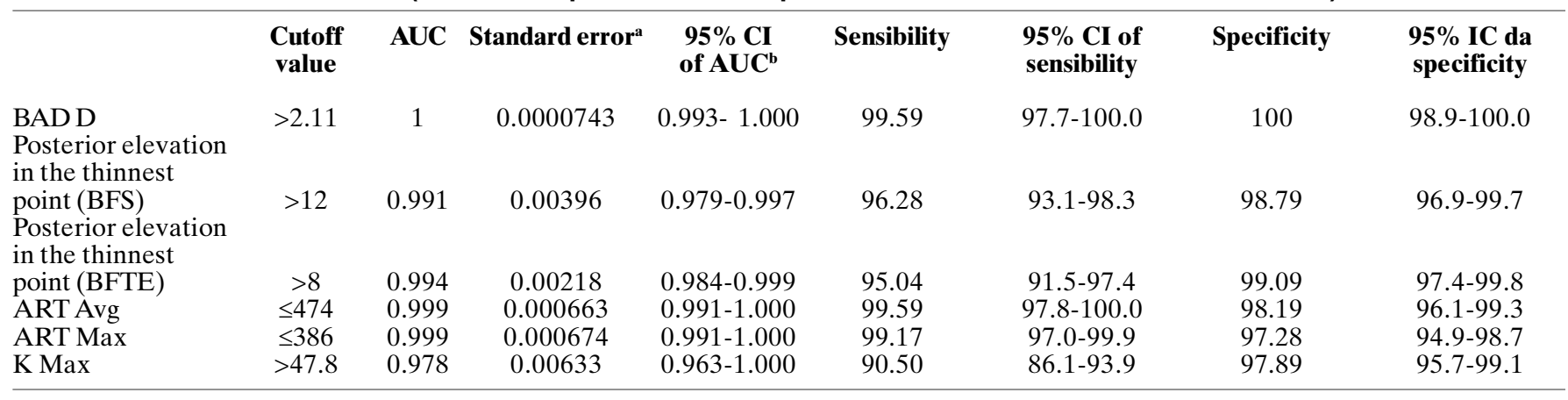

a Method for standard error calculation (DeLong, 1988), listed in the software MedCalc.

b 95\% CI, confidence interval; AUC, area under the curve "receiver operating characteristic".

ART Ave, Ambrósio's Relational Thickness medium; ART Max, Ambrósio's Relational Thickness maximum; BAD-D, D-final value of Belin-Ambrósio Enhanced Ectasia Display; BFS, Best Fit Sphere; BFTE, Best Fit Toric Ellipsoid; K Max, maximum keratometry value; SE, Standard error calculated by the binomial method.

Table 3

Results of the curves "receiver operating characteristic" (ROC) of the parameters of Pentacam (331 normal patients vs. 47 patients with forme fruste keratoconus).

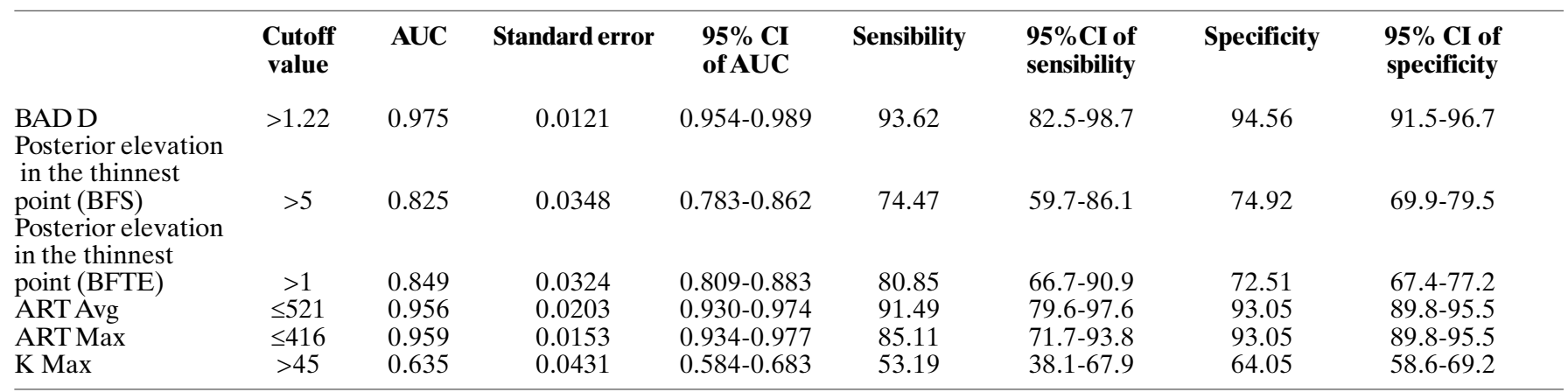

a Method for standard error calculation (DeLong, 1988), listed in the software MedCalc.

b 95\% CI, confidence interval; AUC, area under the curve "receiver operating characteristic".

ART Ave, Ambrósio's Relational Thickness medium; ART Max, Ambrósio's Relational Thickness maximum; BAD-D, D-final value of Belin-Ambrósio Enhanced Ectasia ART Ave, Ambrósio's Relational Thickness medium; ART Max, Ambrósio's Relational Thickness maximum; BAD-D, D-final value of Belin-Ambrósio Enh
Display; BFS, Best Fit Sphere; BFTE, Best Fit Toric Ellipsoid; K Max, maximum keratometry value; SE, Standard error calculated by the binomial method.

between the PPI and the thinnest point.

The "Belin-Ambrósio Enhanced Ectasia Display" (BAD; Figures 4 and 5) allows an overview of tomographic structure of the cornea by combining data from the anterior and posterior elevation, pachymetry and curvature. The BAD considers deviations from normality for different parameters, so that a zero value represents the mean of the normal population and one is the value of a standard deviation value toward the value of the disease (ectasia). ${ }^{3}$ The final ' $\mathrm{D}$ ' is calculated based on the regression analysis, weighing differently the various parameters. Alternatively, Saad and Gatinel developed an efficient method of combining pachymetry and elevation data of Orbscan in discriminant functions to detect keratoconus and forme fruste keratoconus (FFKC)..$^{54}$

Tables 2 and 3 provide the cutting values and the details of curves "receiver operating characteristic" (ROC) of the most 
effective Pentacam parameters for identifying cornea with ectasia. Table 2 refers to a study involving an eye randomly selected from 331 normal patients and 242 patients with bilateral clinical keratoconus. ${ }^{3}$

Interestingly, the fact that the screening of the risk of ectasia should go beyond keratoconus detection is crucial to consider the studies that include mild or subclinical forms of ectasia., ${ }^{3,55}$ One of the most important subgroups consists of eyes with relatively normal topography of keratoconus patients detected in the contralateral eye, being referred as FFKC.,34,56 Table 3 refers to a study which included 47 corneas with FFKC, and the same control group of study ${ }^{57}$ of Table 2. It is critical to adjust the cutoff values to identify such mild cases or those of susceptibility to ectasia. For example, BAD-D has a cutoff value of 2.11 to detect keratoconus (99.59\% sensitivity and $100 \%$ specificity; Table 2 ), but the best cutoff value to detect FFKC is 1.22 (93.62\% sensitivity and $94.56 \%$ specificity). The optimization of the area under the ROC curve can be possible with the cutoff value, but with a minimal and tolerable loss of specificity value. For example, some parameters that are very efficient in the detection of keratoconus, such as the maximum keratometry, may not be useful in identifying cases with FFKC.

Dynamic Scheimpflug imaging to asses corneal deformation

CorVIS ST (Oculus, Wetzlar, Germany) is an NCT with a high-speed Scheimpflug camera that was launched in $2010{ }^{3,29}$ ENREF 28 The coupled Scheimpflug camera covers the horizontal $8 . \overline{5} \mathrm{~mm}$ of the cornea and captures more than 4300 images per second to monitor the response of the cornea to a collimated and calibrated puff of air. The air pulse has a fixed profile with symmetrical configuration and with maximum internal pressure of the pump of $25 \mathrm{kPa} .{ }^{29}$ During the recording time of $30 \mathrm{~ms}, 140$ digital images are acquired with 576 measuring points in each. Advanced algorithms for detecting the cornea contours are applied to each image. The measurement starts with the cornea in its natural convex shape. The puff of air forces the cornea inside (ingoing phase), going through a time of aplannation (ingoing aplannation) in a concavity phase until it reaches its peak. There is a period of oscillation before starting the output or return phase (outgoing phase). The cornea undergoes a second moment of applanation (outgoing applanation) until returning to its natural shape. A possible cam motion can occur in this phase of the measurement. The time and pressure in the first and second applanation moments and when the cornea reaches the maximum point of concavity are recorded. The intraocular pressure (IOP) is based on the deformation data. The amplitude of deformation is detected as the largest displacement of the corneal apex on the image corresponding to the moment of greatest concavity. The radius of curvature in the phase of largest concavity, the lengths and the speeds of the cornea during the applanation phases are also recorded. The lowest value of corneal thickness is also available, and is derived from the first horizontal Scheimpflug image. ${ }^{29}$

Preliminary results have shown that IOP has strong and significant influence on the corneal deformation parameters. In a study involving a model of the eye's anterior chamber composed of hydrophilic contact lenses mounted in a sealed water chamber with adjustable pressure, three lenses with known constitution were evaluated under different pressure levels. ${ }^{58}$ Each different lens showed different deformation amplitude in the pressure levels evaluated, which were greater (less rigid behavior) with lower pressure levels $(\mathrm{P}<0.001$; Bonferroni posthoc test). Interestingly, when evaluated under the same internal pressure, the deformation amplitude demonstrated to be inversely related to the percentage of polymer in the lens composition. However, the thinner lens and with less polymer had an inferior deformation amplitude (more rigid behavior) at higher pressures than the thicker lenses and with more percentage of polymer in lower pressures. Furthermore, the impact of the IOP reduction in the corneal deformation has been well documented in many clinical situations, particularly a case of pressure-induced keratopathy. ${ }^{59}$ Studies comparing normal and keratoconus corneas show statistically significant differences for most of the deformation parameters provided by CorVIS, but with a relatively high overlay between the groups, which limits their diagnostic applications. The combination of parameters using linear discriminant analysis and other techniques of artificial intelligence has been the subject of intense studies by the Brazilian Study Group of Artificial Intelligence and Corneal Analysis (BrAIn). For example, the "Corvis Factor 1" was effective to improve the ability to distinguish normal corneal from those with ectasia, including cases of FFKC $(\mathrm{P}<0.001$, Kruskal-Wallis test with Dunn's post-hoc test). Along with the diagnosis of ectasia, the biomechanical study with the Scheimpflug technology was effective to assess changes after the crosslinking (Roberts, unpublished data, 2011). In the studies conducted at Ohio State University, in the 1st month after the procedure a smaller deformation amplitude ( $P$ $<0.0014)$ was recorded. This result is justified by the increased rigidity of the cornea after the procedure.

The integration between the biomechanical and tomographic data demonstrated a significant improvement in identifying much milder forms of ectasia (Figure 5). This has also to be considered when assessing the risk of progression of ectasia and the prognosis of keratoconus. In a study involving 119 eyes with normal corneas and 19 eyes with FFKC, a combined parameter derived from tomography and biomechanical assessments was designed and presented an area under the ROC curve of 0.999 . This combined parameter had a sensitivity of $100 \%$ and a specificity of $99.2 \%$.

\section{Scheimpflug imae in cataract surgery assisted by}

\section{femtosecond}

Cataract surgery assisted by femtosecond laser depends fundamentally on the imaging of the anterior segment to guide the corneal incisions, the curvilinear and continuous capsulorhexis and the fragmentation of cataracts. ${ }^{33}$ LENSAR Laser System (LENSAR Inc., Winter Park, USA) incorporates a three-dimensional confocal illumination (3D-CSI) consisting of an advanced transmitter of scaning lighting which increases the accuracy of the three-dimensional reconstruction using the Scheimpflug imaging technique. ${ }^{60}$ The system has a lateral (x,y) and longitudinal (z) resolution of less than 10 microns. The ability to determine the position of the anterior and posterior surfaces of the crystalline is increased due to the high-contrast of the optic system on the anatomical edges, which makes the 3D-CSI relatively less sensitive to the dispersion present in higher density cataracts. Additionally, the 3D-CSI provides detailed structural details of cataract, with the potential of automatic classification of their density. Due to the layout of the optical rays, LENSAR can collect biometric data (including rays of anterior and posterior corneal curvature, corneal thickness, anterior chamber depth, anterior and posterior curvature rays of the crystalline and the thickness of the crystalline), also allowing the detection of the crystalline tilt to the optical axis. ${ }^{60}$ Other systems available on the market, and in particular LenSx (Alcon Laboratories, Fort Worth, Texas, USA), Catalys (OptiMedica Corp., California, USA) and Victus Technolas (Bausch \& Lomb/Technolas Perfect Vision $\mathrm{GmbH}$, Germany) use optical coherence tomography (OCT) to assess the location of the intraocular structures. ${ }^{33}$

\section{Conclusion}

The Scheimpflug imaging technique will coexist with other technologies such as OCT and high-frequency ultrasound, but it will have an evolutive role in the area of laser refractive surgery. Continuous advances are expected to strengthen the diagnostic capabilities and surgical planning. Faster computer systems and highresolution cameras also have a significant role in this evolution. Furthermore, artificial intelligence is of key importance in order to increase the safety and efficacy of customized refractive treatments. 


\section{REFERENCES}

1. Wilson SE, Klyce SD. Advances in the analysis of corneal topography. Sury Ophthalmol. 1991;35(4):269-77.

2. Wilson SE, Ambrosio R. Computerized corneal topography and its importance to wavefront technology. Cornea. 2001;20(5):441-54.

3. Ambrosio R, Jr., Nogueira LP, Caldas DL, et al. Evaluation of corneal shape and biomechanics before LASIK. Int Ophthalmol Clin. 2011:51(2):11-38.

Ambrosio R, Jr., Tervo T, Wilson SE. LASIK-associated dry eye and neurotrophic epitheliopathy: pathophysiology and strategies for prevention and treatment. J Refract Surg. 2008;24(4):396-407.

5. Lin DT, Holland S, Tan JC, Moloney G. Clinical results of topography-based customized ablations in highly aberrated eyes and keratoconus/ectasia with cross-linking. J Refract Surg. 2012;28(11):S841-8.

6. Pasquali T and Krueger R. Topography-guided laser refractive surgery. Cur Opin Ophthalmol. 2012;23(4):264-8.

7. Ambrosio R, Jr., Jardim D, Netto MV,Wilson SE. Management of unsuccessfu LASIK surgery. Compr Ophthalmol Update. 2007;8(3):125-41; discussion 1434. Review.

8. Ambrosio R, Jr.,Wilson SE. Complications of laser in situ keratomileusis: etiology, prevention, and treatment. J Refract Surg. 2001;17(3):350-79. Review.

9. Packer M, Fine IH,Hoffman RS. Aspheric intraocular lens selection: the evolution of refractive cataract surgery. Curr Opin Ophthalmol. 2008;19(1):1-4.

10. Packer M, Fine IH, Hoffman RS. Aspheric intraocular lens selection based on corneal wavefront. J Refract Surg. 2009;25(1):12-20.

11. Kanellopoulos AJ, Binder PS. Management of corneal ectasia after LASIK with combined, same-day, topography-guided partial transepithelial PRK an collagen cross-linking: the athens protocol. J Refract Surg. 2010;27(5):323-31.

12. Koller T, Iseli HP, Donitzky C, Ing D, Papadopoulos N, Seiler T. Topography guided surface ablation for forme fruste keratoconus. Ophthalmology. 2006;113(12):2198-202.

13. Reinstein DZ, Archer TJ, Gobbe M. Refractive and topographic errors in topography-guided ablation produced by epithelial compensation predicted by $3 \mathrm{D}$ Artemis VHF digital ultrasound stromal and epithelial thickness mapping. J Refract Surg. 2012;28(9):657-63.

14. Reinstein DZ,Archer TJ, Gobbe M. Combined corneal topography and corneal wavefront data in the treatment of corneal irregularity and refractive error in LASIK or PRK using the Carl Zeiss Meditec MEL 80 and CRS-Master. J Refract Surg. 2009;25(6):503-15.

15. Faria-Correia F, Luz A, Ambrósio Jr R. Managing corneal ectasia prior to keratoplasty. Expert Rev Ophthalmol. 2015;10(1):33-48.

16. Klyce SD. Computer-assisted corneal topography. High-resolution graphic presentation and analysis of keratoscopy. Invest Ophthalmol Vis Sci. 1984;25(12):1426-35.

17. Belin MW, Litoff D, Strods SJ, Winn SS, Smith RS. The PAR Technology Cornea Topography System. Refract Corneal Surg. 1992;8(1):88-96.

18. Belin MW, Khachikian SS. An introduction to understanding elevation-based topography: how elevation data are displayed - a review. Clin Experiment Ophthalmol. 2009;37(1):14-29.

19. Ambrosio R, Jr., Belin MW. Imaging of the cornea: topography vs tomography. J Refract Surg. 2010;26(11):847-9.

20. Maurice DM, Giardini AA. A simple optical apparatus for measuring the corneal thickness, and the average thickness of the human cornea. Br J Ophthalmol. 1951;35(3):169-77.

21. Salz JJ,Azen SP, Berstein J, Caroline P,Villasenor RA, Schanzlin DJ. Evaluation and comparison of sources of variability in the measurement of corneal thickness with ultrasonic and optical pachymeters. Ophthalmic Surg. 1983:14(9):750-4

22. Mandell RB, Polse KA. Keratoconus: spatial variation of corneal thickenss as a diagnostic test. Arch Ophthalmol. 1969;82(2):182-8.

23. Ambrosio R, Jr. Percentage thickness increase and absolute difference from thinnest to describe thickness profile. J Refract Surg. 2010;26(2):84-6; author reply 86-7.

24. Ambrosio R, Jr., Alonso RS, Luz A, Coca Velarde LG. Corneal-thickness spatial profile and corneal-volume distribution: tomographic indices to detect keratoconus. J Cataract Refract Surg. 2006;32(11):1851-9.

25. Salomao MQ, Esposito A, Dupps WJ, Jr. Advances in anterior segment imaging and analysis. Curr Opin Ophthalmol. 2009;20(4):324-32.

26. Roberts C. Biomechanical customization: the next generation of laser refractive surgery. J Cataract Refract Surg. 2005;31(1):2-5.

27. Luce DA. Determining in vivo biomechanical properties of the cornea with an ocular response analyzer. J Cataract Refract Surg. 2005;31(1):156-62.

28. Dupps WJ, Jr., Wilson SE. Biomechanics and wound healing in the cornea. Exp Eye Res. 2006;83(4):709-20.

29. Ambrosio R, Jr., Ramos I, Luz A, et al. Dynamic Ultra-High Speed Scheimpflug Imaging for assessing corneal biomechanical properties. Rev Bras Oftalmol. 2013;72(2):99-102.

30. Dorronsoro C, Pascual D, Perez-Merino P, Kling S, Marcos S. Dynamic OCT measurement of corneal deformation by an air puff in normal and cross-linked corneas. Biomed Opt Express. 2012;3(3):473-87.

31. Schumacher S, Fromm M, Oberheide U, Gerten G, Wegener A, Lubatschowski $H$. In vivo application and imaging of intralenticular femtosecond laser pulses for the restoration of accommodation. J Refract Surg. 2008;24(9):991-5.

32. Palanker DV, Blumenkranz MS, Andersen D, et al. Femtosecond laser-assisted cataract surgery with integrated optical coherence tomography. Sci Transl Med. 2010;2(58):58ra85.
33. Roberts TV, Lawless M, Chan CC, et al. Femtosecond laser cataract surgery: technology and clinical practice. Clin Experiment Ophthalmol. 2013;41(2):180-6. Wegener A, Laser-Junga $H$. Photography of the anterior eye segment according to Scheimpflug's principle: options and limitations - a review. Clin Experiment Ophthalmol. 2009:37(1):144-54.

35. Foo KP, Maclean H. Measured changes in cataract over six months: sensitivity of the Nidek EAS-1000. Ophthalmic Res. 1996;28 Suppl 2: 32-6.

36. Wegener A, Hockwin O, Laser H and Strack C. Comparison of the Nidek EAS 1000 system and the Topcon SL-45 in clinical application. Ophthalmic Res 1992;24 Suppl 1:55-62.

37. Baez KA, Orengo S, Gandham S, Spaeth GL. Intraobserver and interobserver reproducibility of the Nidek EAS-1000 Anterior Eye Segment Analysis System. Ophthalmic Surg. 1992;23(6):426-8.

38. Cairns G,McGhee CN, Collins MJ, Owens Hand Gamble GD. Accuracy of Orbscan II slit-scanning elevation topography. J Cataract Refract Surg. 2002;28(12):2181-7.

39. Cairns G, McGhee CN. Orbscan computerized topography: attributes, applications, and limitations. J Cataract Refract Surg. 2005;31(1):205-20.

40. Kim H, Joo CK. Measure of keratoconus progression using Orbscan II. J Refract Surg. Jo0 CK. Measure

41. Auffarth GU,Tetz MR, Biazid Y Volcker HE Measuring anterior chamber depth with Orbscan Topography System. J Cataract Refract Surg. 1997;23(9):1351-5.

42. Auffarth GU, Wang L, Volcker HE. Keratoconus evaluation using the Orbscan Topography System. J Cataract Refract Surg. 2000;26(2):222-8.

43. Belin MW and Khachikian SS. New devices and clinical implications for measuring corneal thickness. Clin Experiment Ophthalmol. 2006;34(8):729-31.

44. Belin MW, Khachikian SS, McGhee CN, Patel D. New technology in cornea imaging. Int Ophthalmol Clin. 2010;50(3):177-89.

45. Aptel F, Chiquet C, Beccat S, Denis P. Biometric evaluation of anterior chamber changes after physiologic pupil dilation using Pentacam and anterior segment optical coherence tomography. Invest Ophthalmol Vis Sci. 2012:53(7):4005-10.

46. Rao SN, Raviv T, Majmudar PA, Epstein RJ. Role of Orbscan II in screening keratoconus suspects before refractive corneal surgery. Ophthalmology. 2002;109(9):1642-6.

47. Lim L, Wei RH, Chan WK, Tan DT. Evaluation of keratoconus in Asians: role of Orbscan II and Tomey TMS-2 corneal topography. Am J Ophthalmol. 2007; 143(3):390-400.

48. Belin MW, Asota IM, Ambrosio R, Jr., Khachikian SS. What's in a name: keratoconus, pellucid marginal degeneration, and related thinning disorders. Am J Ophthalmol. 2011;152(2):157-62 e1

49. Tummanapalli SS, Maseedupally V, Mandathara P, Rathi VM, Sangwan VS Evaluation of corneal elevation and thickness indices in pellucid marginal degeneration and keratoconus. J Cataract Refract Surg. 2013;39(1):56-65.

50. Faria-Correia F, Ramos IC, Lopes B, et al. Topometric and Tomographic Indices for the Diagnosis of Keratoconus. Int J Kerat Ect Cor Dis. 2012;1(2):92-9.

51. Luz A, Ursulio M, Castaneda D, Ambrosio R, Jr. [Corneal thickness progression from the thinnest point to the limbus: study based on a normal and a keratoconus population to create reference values]. Arq Bras Oftalmol. 2006;69(4):57983. Portuguese

52. Ambrosio R, Jr., Caiado AL, Guerra FP, et al Novel pachymetric parameter based on corneal tomography for diagnosing keratoconus. J Refract Surg. 2011;27(10):753-8.

53. Buhren J, Kook D, Yoon G, Kohnen T. Detection of subclinical keratoconus by using corneal anterior and posterior surface aberrations and thickness spatial profiles. Invest Ophthalmol Vis Sci. 2010;51(7):3424-32

54. Saad A, Gatinel D. Topographic and tomographic properties of forme fruste keratoconus corneas. Invest Ophthalmol Vis Sci. 2010;51(11):5546-55.

55. Ambrosio R, Jr., Dawson DG, Salomao M, Guerra FP, Caiado AL, Belin MW Corneal ectasia after LASIK despite low preoperative risk: tomographic and biomechanical findings in the unoperated, stable, fellow eye. J Refract Surg. 2010;26(11):906-11.

56. Klyce SD. Chasing the suspect: keratoconus. Br J Ophthalmol. 2009;93(7):845-7.

57. Ambrosio R, Jr., Valbon BF, Faria-Correia F, Ramos I, Luz A. Scheimpflug imaging for laser refractive surgery. Curr Opin Ophthalmol. 2013;24(4):310-20.

58. Correia FF, Ramos I, Roberts CJ, Steinmueller A, Krug M, Ambrosio R, Jr Impact of chamber pressure and material properties on the deformation response of corneal models measured by dynamic ultra-high-speed Scheimpflug response of corneal models measured by dynamic

59. Faria-Correia F, Ramos I, Valbon B Luz A, Roberts CJ, Ambrosio R, Jr. Scheimpflug-based tomography and biomechanical assessment in pressureinduced stromal keratopathy. J Refract Surg. 2013;29(5):356-8.

60. Packer M, Klyce SD, Smith C. The LENSAR Laser System-fs 3D for Femtoseond Cataract Surgery. US Ophthalmic Review. 2014;7(2):89-94.

\section{Corresponding author:}

Renato Ambrósio Jr, MD, PhD - Instituto de Olhos Renato Ambrósio

Rua Conde de Bonfim 211 / 712 - 20520 - 050

Phone: 5521 2234-4233

E-mail: dr.renatoambrosio@gmail.com 